\title{
CAPÍTULO 22: COLÁGENO, GELATINA E SUAS APLICAÇÕES NA INDÚSTRIA ALIMENTÍCIA: UMA VISÃO GERAL FOCANDO NA MATÉRIA-PRIMA DA PISCICULTURA
}

\section{CHAPTER 22: COLLAGEN, GELATIN AND ITS APPLICATIONS IN THE FOOD INDUSTRY: AN OVERVIEW FOCUSING ON THE RAW MATERIAL OF FISH FARMING}

\author{
Jessica Costa da Silva ${ }^{1}$; Beatriz de Aquino Marques da Costa ${ }^{2}$; Luiz Henrique Svintistkas Lino ${ }^{3}$; \\ Vagne de Melo Oliveira ${ }^{4}$ Ana Lúcia Figueiredo Porto ${ }^{5}$
}

\begin{abstract}
Resumo
A piscicultura tem sido um dos setores mais desenvolvidos da aquicultura, todavia, a alta demanda de produção de peixes tem gerado grande quantidade de resíduos (pele, ossos, escamas), os quais agregam valor comercial e pode ser reaproveitado para extração de biomoléculas, entre elas, o colágeno, proteína bastante abundante em mamíferos e que a partir da sua hidrólise parcial é possível obter outro biopolímero, a gelatina. Esta revisão de literatura objetivou investigar o reaproveitamento de resíduos de peixe para produção de gelatina e suas aplicações na indústria de alimentos. Para tanto, foram acessadas diferentes plataformas utilizando palavras-chaves em inglês e português, priorizando publicações de 2016 a 2020. A pele de peixe tem sido utilizada para a produção de gelatina, a qual apresenta estrutura sólida, gelificante e intumescente, características de interesse dos segmentos farmacêuticos, cosméticos e nutracêuticas, entre outros. Além disso, possui grande vantagem comparada a de mamíferos, que podem oferecer riscos à saúde pela transmissão de doenças, além das restrições religiosas quanto a sua utilização. Conclui-se que os subprodutos de peixe têm se mostrado bastante proveitosos na produção de gelatina, assim como, sua aplicação na indústria de alimentos por ser uma fonte segura e não transmitir doenças.
\end{abstract}

Palavras-Chave: Alimentos, Aquicultura, Biopolímero, Produto.

\begin{abstract}
Fish farming has been one of the most developed sectors of aquaculture, however, the high demand for fish production has generated a large amount of waste (skin, bones, scales), which add commercial value and can be reused for the extraction of biomolecules, among them, collagen, a protein abundant in mammals and that from its partial hydrolysis it is possible to obtain another biopolymer, gelatin. This literature review aimed to investigate the reuse of fish waste for gelatin production and its applications in the food industry. To this end, different platforms were accessed using keywords in English and Portuguese, prioritizing publications from 2016 to 2020. Fish skin has been used for the production of gelatin, which has a solid, gelling and intumescent structure, characteristics of interest to pharmaceutical, cosmetic and nutraceutical segments, among others. In addition, it has a great advantage compared to mammals, which can offer health risks due to the transmission of diseases, in addition to religious restrictions regarding their use. It is concluded that fish by-products have

\footnotetext{
${ }^{1}$ Doutoranda em Ciências Biológicas, Universidade Federal de Pernambuco, jess.cost15@gmail.com

${ }^{2}$ Mestranda em Biociência Animal, Universidade Federal Rural de Pernambuco, deaquinobeatriz@gmail.com

${ }^{3}$ Mestrando em Biologia Aplicada a Saúde, luiz.svintiskas@ @otmail.com

${ }^{4}$ Doutor, Universidade Federal Rural de Pernambuco, vagne melo@hotmail.com

${ }^{5}$ Professora Titular, Universidade Federal Rural de Pernambuco, vagne melo@ hotmail.com
} 
been shown to be quite useful in the production of gelatin, as well as its application in the food industry as it is a safe source and does not transmit diseases.

Keywords: Food, Aquaculture, Biopolymer, Product.

\section{Introdução}

O colágeno e a gelatina de peixes são de interesse crescente. A estrutura do colágeno foi estudada usando várias tecnologias modernas e pode ser observada uma variação de acordo com as fontes e as estações do ano, o que pode afetar suas aplicações e condições ideais de extração. Numerosos estudos investigaram as bioatividades e os efeitos biológicos do colágeno, gelatina e seus peptídeos de hidrólise, usando modelos de ensaio in vitro e in vivo. Além do valor nutricional estabelecido como fonte de proteína, colágeno e produtos derivados de colágeno podem exercer várias atividades biológicas em potencial nas células da matriz extracelular através dos peptídeos derivados de alimentos correspondentes após a ingestão, e isso pode justificar suas aplicações em suplementos alimentares e preparações farmacêuticas. Além disso, um número crescente de novas aplicações foi encontrado para colágeno e gelatina (LIU et al., 2015).

A gelatina é um ingrediente multifuncional usado em alimentos, produtos farmacêuticos, cosméticos e filmes fotográficos como agente gelificante, estabilizador, espessante, emulsificante e formador de filme. Como um hidrocolóide termorreversível com um intervalo mais estreito entre as temperaturas de fusão e gelificação, ambas abaixo da temperatura do corpo humano, a gelatina oferece vantagens exclusivas sobre os agentes gelificantes à base de carboidratos (BORAN; REGENSTEIN, 2010).

A gelatina proveniente de peixes tem se destacado por trazer benefícios extraordinários para pessoas com doenças crônicas, como diabetes, hipertensão e osteoporose (LV et al., 2019), além de poder substituir a gelatina de mamíferos (MIRZAPOURKOUHDASHT et al., 2020).

Dentre as espécies de peixes com potencial para fornecer colágeno para produção de gelatina, a tilápia-do-Nilo (Oreochromis niloticus) merece especial atenção devida ser a espécie que lidera a produção na piscicultura nacional e, consequentemente, liberando grande volume de pele para reutilização, extração e produção de gelatina.

Assim, dentro desse contexto, esta revisão objetivou investigar o reaproveitamento de resíduos de peixes para a produção de gelatina, priorizando sua aplicação na indústria de alimentos e bebidas. 


\section{Desenvolvimento}

Para esta mini-revisão bibliográfica, foram realizadas buscas em base de dadoscientíficos, priorizando publicações entre 2016 e 2020, tais como: ScienceDirect (http://www.sciencedirect.com/), PubMed (https://pubmed.ncbi.nlm.nih.gov/), Scielo (http://www.scielo.com/) e Google acadêmico (https://scholar.google.com.br/), utilizando as palavras-chaves: colágeno, gelatina de peixe, benefícios da gelatina de peixe, propriedades reológicas da gelatina de peixe. Não houve nenhuma restrição quanto ao tipo e artigo (pesquisa, revisão, nota científica, dissertação e/ou teses de doutoramento). O conteúdo foi identificado, selecionado e organizado para formulação dos conceitos, de acordo com a conveniência dos autores.

\subsection{Piscicultura, colágeno e gelatina}

O crescimento da indústria da Pesca e Aquicultura tem sido bastante promissor para a economia mundial. De acordo com os dados fornecidos pela Organização das Nações Unidas para Agricultura e Alimentação (FAO), em 2018 o total da produção mundial da pesca capturada foi a mais alta registrada, atingindo 96,4 milhões de toneladas, um aumento significativo de 5,4\% em relação à média dos anos anteriores (FAO, 2018).

No Brasil, o crescimento da piscicultura tem se destacado para atender a demanda de alimentos por ser uma fonte rica em proteínas, aminoácidos e ômega-3 (OLIVEIRA et al., 2017a), auxiliando na alimentação balanceada e saudável, para manter o bom funcionamento celular e de todos os órgãos presentes no corpo (STOHS; KITCHENS, 2019).

Devido ao alto consumo desse tipo de produto, os pescadores artesanais e a indústria pesqueira têm gerado muitos resíduos durante as etapas de processamento/beneficiamento, pois aproximadamente $70 \%$ do peso dos peixes são constituídos por resíduos, tais como: ossos, pele, escamas, barbatanas, nadadeiras e vísceras internas, como fígado, estômago e intestino, os quais quase sempre são descartados inadequadamente no ambiente de coleta ou lixeiras, tornando-o bastante danoso ao meio ambiente (OLIVEIRA et al., 2017b).

De potencial ilimitado, os subprodutos descartados do beneficiando de peixes são fontes de biomoléculas proteicas de grande interesse comercial, como o colágeno (SUN et al., 2017). O colágeno é uma proteína fibrosa e insolúvel, sendo a principal estrutura dos tecidos conjuntivos, estando presente em tendões, pele, ligamentos, ossos, entre outros (ALVES et al., 2017; RIAZ et al., 2018). 
O colágeno tem sido utilizado em inúmeras aplicações visando melhorias a saúde humana e animal, através do seu uso em processos de cicatrização, tratamento de ferimentos e queimaduras (LIMA-JÚNIOR et al., 2017; ARUMUGAM et al., 2018). Ainda, tem sido empregado na indústria alimentícia, principalmente, para a produção de gelatina (PANG et al., 2017), na indústria de couro para confecção de bolsas e sapatos, e no segmento cosmético para proteção da pele e antienvelhecimento (ALVES et al., 2017; OLIVEIRA et al., 2017b). Até o momento, já foram descritos na literatura 29 subtipos diferentes de moléculas de colágeno, sendo mais abundante o colágeno Tipo I (OLIVEIRA et al., 2017a), que além dos peixes, pode ser extraído de vários organismos de vida marinha como: lula, polvo, esponjas do mar, camarão (TAYEL et al., 2018), entre outros.

A partir da hidrólise do colágeno pela colagenase, uma metaloproteínase que cliva a tripla hélice dessa proteína, são produzidos fragmentos peptídicos menos, em tamanho e massa molecular, os chamados "peptídeos" (OLIVEIRA et al., 2017B). Esses peptídeos têm sido amplamente empregados em suplementos alimentares pela sua potencialidade nutracêutica, biomédica e farmacêutica, principalmente, visando a prevenção e tratamento de doenças ósseas, como osteoporose e osteoartrite (PORFIRIO et al., FANARO, 2016; KWATRA, 2020), implantes regenerativos de córnea (JANGAMREDDY et al., 2018) e até na regeneração da pele (ZHANG et al., 2020). Através do colágeno, podem ser produzidos vários tipos de gelatina com multifuncionalidades de interesse dentro do segmento alimentício e de bebidas (LIN et al., 2017; RENUKA et al., 2019).

As principais fontes de gelatina são mamíferos e peixes (Figura 1). A gelatina comercial é produzida a partir de pele de suínos e do couro e ossos de bovinos (LIU et al., 2019). Recentemente, algumas matérias-primas alternativas chamaram a atenção de pesquisadores e da indústria, não apenas porque superam as preocupações religiosas compartilhadas por judeus e muçulmanos, mas também porque fornecem, em alguns casos, vantagens tecnológicas sobre as gelatinas de mamíferos (BORAN; REGENSTEIN, 2010).

As peles de peixes de várias espécies de peixes estão entre as outras fontes que foram amplamente estudadas como fontes para a produção de gelatina, com potencial significativo para a produção de gelatina de alta qualidade com diferentes temperaturas de fusão e gelificação em uma faixa muito mais ampla do que as gelatinas de mamíferos, mas ainda assim têm uma força e viscosidade de gel suficientemente alta (BORAN; REGENSTEIN, 2010).

Para ser consumida, a gelatina passa por processos de purificação para melhor uso industrial, principalmente na área de alimentos. No entanto, a aceitabilidade dos produtos de 
gelatina depende muito das fontes animais da gelatina. As gelatinas extraídas de fontes suínas e bovinas têm características atraentes, mas aceitação limitada devido a proibições religiosas e possíveis ameaças zoonóticas, enquanto a gelatina de peixe é bem-vinda em todas as religiões e culturas (ALI et al., 2018).

Figura 1. Principais fontes de colágeno e gelatina de origem comercial.
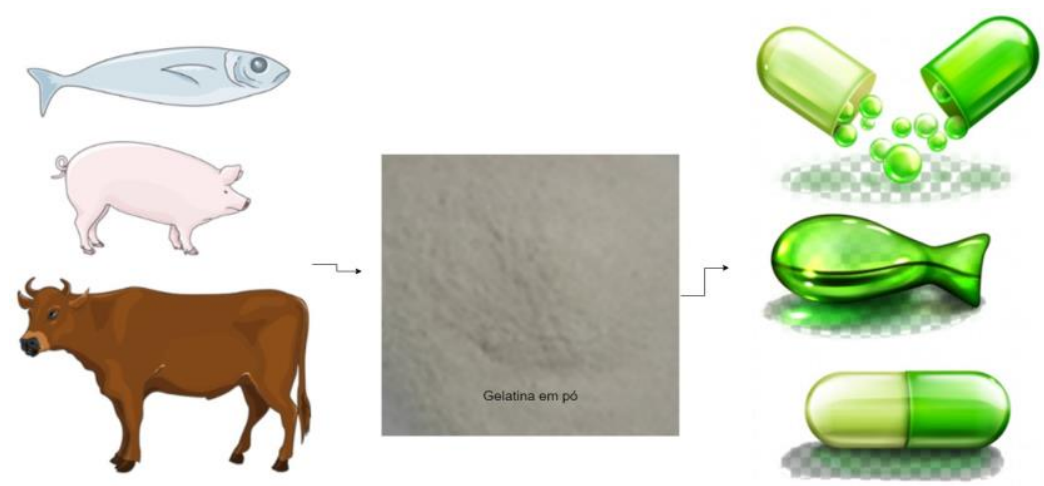

Fonte: Imagens de SMART - Servier Medical Art (2020) e Freepik (2020)

\subsection{Obtenção de proteínas colagenosas}

Para que haja a produção de gelatina é preciso, primeiramente, extrair o colágeno. Para obtenção das proteínas colagenosas, a pele de peixe é submetida a várias etapas de tratamento seguindo protocolos rigorosos (Figura 2) durante a realização de análises físicoquímicas para a confirmação do colágeno extraído.

Figura 2. Processo de extração do colágeno.

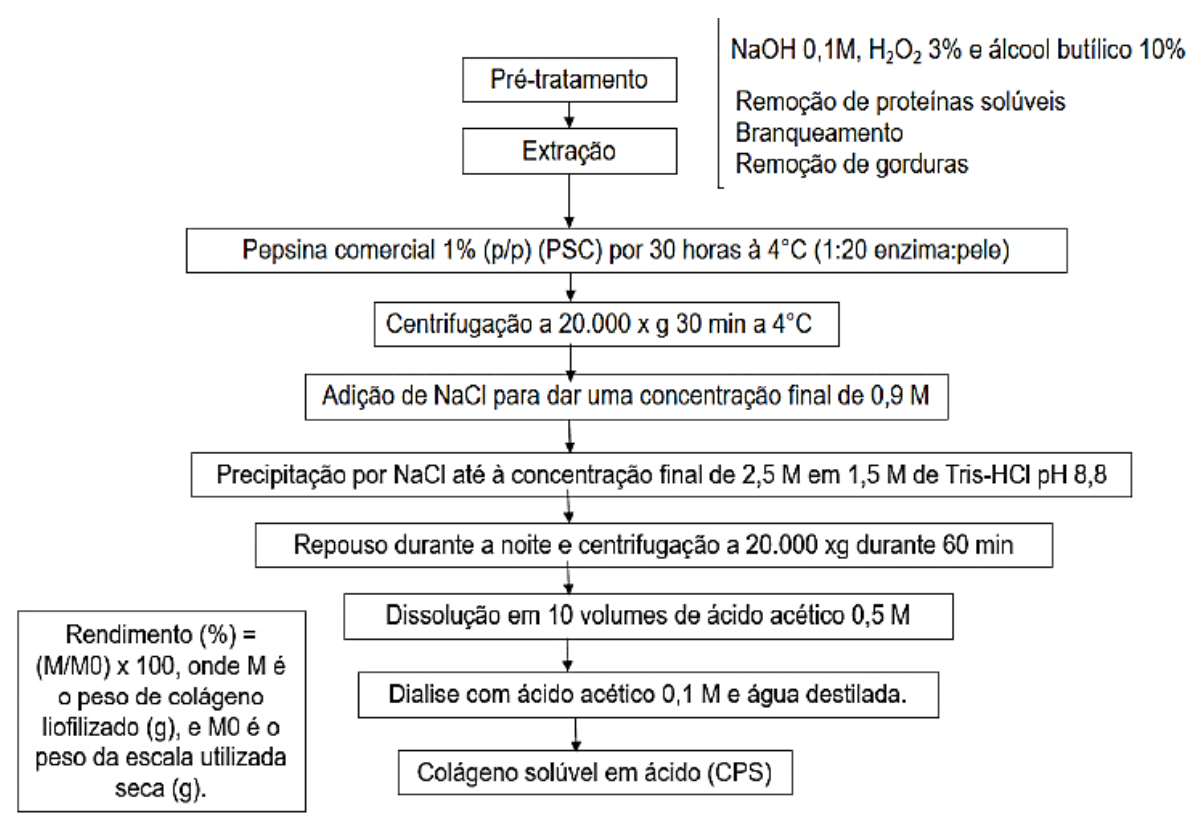

Fonte: Oliveira et al.(2017a). Imagem cedida gentilmente pelos autores. 


\subsection{Produção e composição de gelatinas}

A gelatina é conhecida como um pó, apresentando estrutura firme, sólida, gelificante, e intumescente (MIRZAPOUR-KOUHDASHT et al., 2020), que ao entrar contato com a água dissolve facilmente, principalmente após a desnaturação por calor (ABDELHEDI et al., 2017). A composição dos aminoácidos da gelatina vai variar de acordo com a fonte extraída, isso envolve as espécies, as formas de pré-tratamento e, principalmente, os tipos extração (BENJAKUL; KITTIPHATTANABAWON, 2018).

A gelatina é obtida através da hidrólise do colágeno, podendo ser extraída por vários métodos, entre eles estão os dois tipos de processo mais realizados para a sua produção. A gelatina tipo A (ponto isoelétrico: 6-9) é extraída por processos ácidos a partir de, por exemplo, matéria-prima de peixes e suínos, pelo fato destas não possuírem grandes quantidades de ligações químicas, o que torna o processo mais rápido por não exigir prétratamento intensivo e longo; A gelatina tipo B (ponto isoelétrico 5), é extraído através de processos alcalinos, utilizando, por exemplo, matéria-prima proveniente de bovinos (ossos, pele e tendão). Nesse processo o pré-tratamento é intenso devido à grande quantidade de ligações químicas (SILVA, 2016). A qualidade da gelatina é determinada industrialmente pela resistência do gel, viscosidade, temperaturas de fusão ou gelificação, conteúdo de água e segurança microbiológica. Para os fabricantes de gelatina, o rendimento de uma determinada matéria-prima também é importante (BORAN; REGENSTEIN, 2010). Algumas das propriedades desejáveis para uma boa gelatina comercial estão ilustradas na Figura 3.

Figura 3. Propriedades desejadas numa gelatina comercial

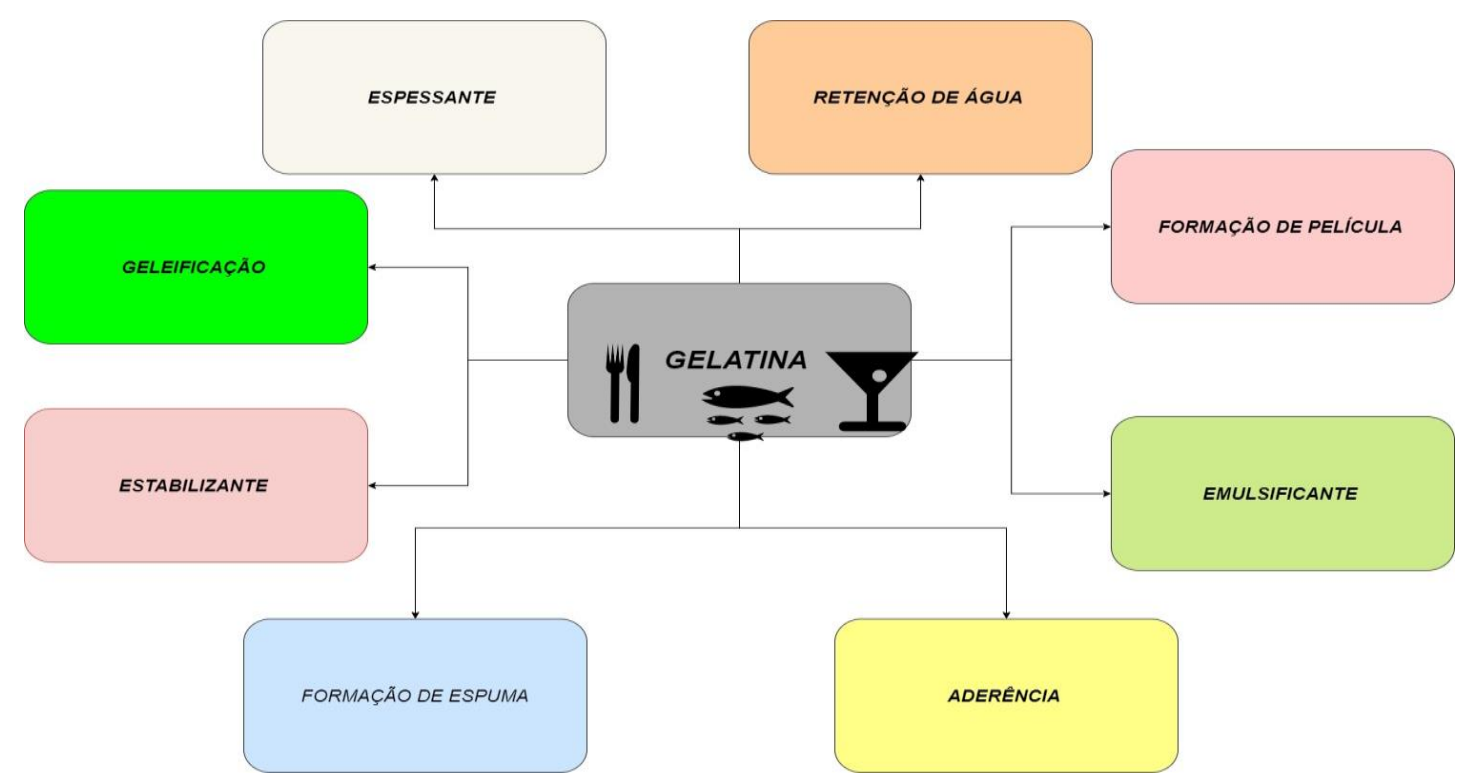

Fonte: Rousselot ${ }^{\circledR}$ (2020). Montagem realizada através do software Diagram. 


\subsection{Produção e caracterização da gelatina de peixe}

O processo para extração de gelatina exige várias etapas: pré-tratamento da matériaprima, extração, purificação, concentração, secagem, moagem, peneiramento e mistura. Após esse processo a gelatina pode ser submetida a diversos tipos de caracterização quanto ao teor de proteínas, determinação da umidade, teor de conteúdo mineral (cinzas), pH e acidez titulável (SILVA et al., 2018).

A qualidade da gelatina está associada às suas propriedades reológicas (Tabela 1), ou seja, verificando a sua elasticidade, viscosidade, ponto de fusão e força (HUANG et al., 2017), sendo a viscosidade e a força do gel algumas das propriedades reológicas mais importantes para a comercialização da gelatina (LIN et al., 2017), tendo em vista que essa caracterização física varia em cada fonte de matéria-prima.

Tabela 1. Estudos sobre as propriedades reológicas com diferentes tipos de aplicações na gelatina de peixe.

\begin{tabular}{ccc}
\hline Nome científico & Estudo & Autor/ano \\
\hline Hypophthalmichthys nobilis & Efeito de Fosforilação e gelificação & Zhang et al. (2017) \\
Ctenopharyngodon idellus & Modelo para cremes de café & Huang et al. (2020) \\
Hamiramphus far & Potencial de revestimento & Abdelhedi et al. (2017) \\
Hypophthalmichthys nobilis & Enzima modificada através gelatina de peixe & Huang et al. (2017) \\
Produto comercial (tilápia) & Efeito da K-carragena da estrutura da gelatina & Sow et al. (2017) \\
Oncorhynchus mykiss & Efeito dos solutos de Confeitaria & Kamer et al. (2019) \\
Produto comercial (tilápia) & Efeito da propriedade antioxidante do gel de gelatina & Sinthusamran et al. (2018) \\
\hline
\end{tabular}

Fonte: Acervo pessoal

Estudos realizados por Huang et al. (2018) apresentaram várias técnicas reológicas associadas a textura e microestrutura para melhor compreensão do efeito de fosforilação nas propriedades de gelificação e emulsificação da gelatina de peixes, sendo também analisadas as propriedades cinéticas, viscosidade aparente, varredura de temperatura e frequência, análise do perfil de textura, aspecto microscópico, atividades emulsificantes e a força atômica microscópica.

A gelatina pode ser extraída tanto de peixes de água fria, quanto em peixes de água morna (SULTANA et al., 2018), sendo assim, os subprodutos de peixes provenientes de água morna não tem apresentado resultados muito satisfatórios quanto às suas propriedades reológicas, diferentemente, dos subprodutos dos peixes de água fria, que têm passado por diferentes modificações em seus protocolos com o intuito de aumentar o desenvolvimento de suas aplicações na indústria (CHUNG, 2020). No entanto, vê-se que a avaliação da textura e 
as propriedades reológicas tem sido importante, sobretudo, para a indústria de alimentos, um exemplo, confeitaria (ALTAN KANER et al., 2018).

\subsection{Vantagens da produção de gelatina de peixe}

O uso da gelatina de peixe tem sido vantajosa para aplicação industrial por apresentar características semelhantes à gelatina de mamíferos, muito devido à ausência de restrições sanitárias e socioculturais (ABDELHEDI et al., 2017; SULTANA et al., 2017).

$\mathrm{Na}$ questão sanitária, existem preocupações a respeito das gelatinas provenientes de fontes bovinas e suínas devido a possível transmissão de enfermidades (LAURINDO et al., 2017), como a Encefalopatia Espongiforme Bovina (PANG et al., 2017; SILA et al., 2017; MIRZAPOUR-KOUHDASHT et al., 2020), uma doença degenerativa crônica que afeta os Sistema Nervoso Central (SNC) (LAURINDO et al., 2017).

Estudos realizados por Pang et al. (2017) relataram que a diferença entre as gelatinas é a menor quantidade de prolina (Pro) na gelatina proveniente de mamíferos, enquanto na gelatina de peixe é menor a quantidade de hidroxiprolina (Hyp). Ainda, os autores reforçam que a gelatina bovina pode ser substituída pela gelatina de tilápia em iogurte com baixo teor de gordura, tendo em vista que ambas apresentam características semelhantes. Além disso, o hidrolisado da gelatina de peixe desempenha amplas funções biológicas, como antidiabética, anti-hipertensiva, crioprotetora, antitumoral, antioxidante (KARAYANNAKIDIS\& ZOTOS, 2016; LV et al., 2019).

Já foi reportado com sucesso o uso da gelatina de peixes dulcícolas com potencial para uso industrial, além da tilápia, a gelatina produzida da carpa (Cyprinus carpio) a apresentou bom potencial antioxidante (TKACZEWSKA; BUKOWSKI; MAK, 2018). Todavia, a aplicação da gelatina proveniente de peixes no mercado comercial ainda é baixa, quando comparada a gelatina de mamíferos (LIN et al., 2017).

\subsection{Produção de gelatina com pele de peixe}

A pele de peixe tem sido um dos principais resíduos utilizados na indústria do processamento de pescado e frutos do mar (ABDLHEDI et al., 2017; SULTANA et al., 2018). Embora alguns estudos registrem a produção de gelatina proveniente de outros tipos de resíduos, por exemplo, a escama de carpa-comum, Cyprinus carpio (HUANG et al., 2018), a gelatina proveniente da pele tem apresentado maior rendimento e consequentemente, é a mais aplicada para a elaboração de produtos na indústria. Através da Tabela 2 é possível identificar 
que a variedade de espécies aquáticas já registradas na literatura utilizadas para a extração de colágeno e produção de gelatina para fins biotecnológicos.

Tabela 2. Espécies de peixes fontes de gelatinas extraídas de subprodutos de pele.

\begin{tabular}{|c|c|}
\hline Nome científico & Autor \\
\hline Oreochromis niloticus & Zhang et al. (2016) \\
\hline Ictalurus punctatus & Zhang et al. (2016) \\
\hline Liza aurata & Bkhairia et al. (2016) \\
\hline Anguilla anguilla & Sila et al. (2017) \\
\hline Hemiramphus far & Abdelhedi et al.(2017) \\
\hline Ctenopharyngodon idellus & Cai et al. (2017) \\
\hline Hypophthalmichthys nobilis & Huang et al. (2017) \\
\hline Oncorhynchus mykiss & Cheunget al. (2017) \\
\hline Probarbus jullieni & Ali et al. (2018) \\
\hline Cyprinus carpio & Santos et al. (2018) \\
\hline Brycon amazonicus & Silva et al. (2018) \\
\hline Colossoma macroponum & Silva et al. (2018) \\
\hline Katsuwonus pelamis & AksunTümerkan et al. (2019) \\
\hline Aluterus monoceros & Renukaetal. (2019) \\
\hline Epinephelus diacanthus & Renuka et al. (2019) \\
\hline Salmo salar & Pępczyńska et al. (2019) \\
\hline Oreochromis aureus & Sow et al. (2019) \\
\hline Pollachius virens & Casanova et al. (2020) \\
\hline Oreochromis mossambicus & Tan et al. (2020) \\
\hline
\end{tabular}

Fonte: Dados da pesquisa (2020).

\subsection{Gelatina de peixe na produção de alimentos}

$\mathrm{Na}$ indústria de alimentos, a gelatina tem sido amplamente utilizada na preparação de molhos, assados, sobremesas, geleias, doces, carnes e produtos com baixo teor de gordura (GHORANI et al., 2020). Com essa grande variedade de produtos, a indústria tem buscado diferentes fontes de matéria-prima, com o intuito de expandir a sua aplicação e fornecer produtos de excelente qualidade para a sociedade. 
A gelatina de peixe tem apresentado resultados bastante satisfatórios em suas aplicações, e por isso se tornou um dos biopolímeros mais utilizados na produção de filmes e embalagens biodegradáveis (SYAHIDA et al., 2020), podendo ser usada como espuma emulsificante intumescente devido a sua propriedade tensoativa (HUANG et al., 2017), agente microencapsulante devido as suas propriedades reológicas (RENUKA et al., 2019), material para produção de cápsulas (LIU et al., 2018), emulsificantes para a produção de óleo em água, por apresentar excelente biocompatibilidade e biodegrabilidade (ZHANG et al., 2020).

Dentre as comprovações do uso da gelatina de fontes aquáticas, tem sido relatado que emulsões de peixes estabilizadas com gelatina podem ser bastante inovadoras em bebidas e alimentos (ZHANG et al., 2020), além apresentar potencial na indústria de confeitaria para a produção de gomosos, exemplo, balas, conhecido, popularmente como confeitos (ALTAN KAMER et al., 2018).Com o avanço da tecnologia, a gelatina de peixe tem ganhado força em suas aplicações fazendo com que sejam valorizadas tanto quanto a gelatina de mamíferos, tornando-se também uma excelente alternativa para substituição da gelatina bovina devido as suas propriedades funcionais (HUANG et al., 2020).

\section{Considerações finais}

A gelatina de peixe tem se tornado cada vez mais valorizada, principalmente por sua fonte de matéria-prima (resíduos, rejeitos e/ou subprodutos) não possuírem necessidade de grandes investimentos. Além disso, tem sido uma alternativa bastante promissora por não oferecer nenhum risco à saúde humana, podendo ser uma substituta da gelatina de mamíferos e diminuir a quantidade de resíduos sólidos que são descartados inadequadamente no meio ambiente, contribuindo ainda para o aumento da renda e margem de lucro.

\section{Referências}

ABDELHEDI, O.; NASRI, R.; MORA,L.; TOLDRÁ, F.; NASRI, M.; JRIDI, M. Collagenous proteins from black-barred halfbeak skin as a source of gelatin and bioactive peptides. Food Hydrocolloids, v.70, p.123-133, 2017.

ABDELHEDI, O.; JRIDI, M.; NASRI, R.; MORA, L.; TOLDRÁ, F.; NASRI, M.. Rheological and structural properties of Hemiramphus far skin gelatin: Potential use as an active fish coating agent. Food Hydrocolloids, v. 87, p. 331-34, 2019.

AKSUN TÜMERKAN, E.T.; CANSU, Ü.; BORAN, G.; REGENSTEIN, J.M.; ÖZOĞUL, F. Physiochemical and functional properties of gelatin obtained from tuna, frog and chicken skins. Food Chemistry, v. 287, p. 273-279, 2019. 
ALI, A.M.M.; KISHIMURA, H.; BENJAKUL, S. Physicochemical and molecular properties of gelatin from skin of golden carp (Probarbus jullieni) as influenced by acid pretreatment and prior-ultrasonication. Food Hydrocolloids, v. 82, 9. 164-172, 2018.

ALI, E.; SULTANA, S.; HAMID, S.; HOSSAIN, M.; YEHYA, W.A.; KADER, A.; BHARGAVA, S.K. Gelatin controversies in food, pharmaceuticals, and personal care products: Authentication methods, current status, and future challenges. Critical reviews in food science and nutrition, v.58, n.9, p.1495-1511, 2018.

ALTAN KAMER, D.D.; PALABIYIK, I.; ISIK, N.O.; AKYUZ, F.; DEMERCI, A.S.; GUMUS, T. Effect of confectionery solutes on the rheologicalproperties of the fish (Oncorhynchus mykiss) gelatin. LWT, v. 101, p. 499-505, 2018.

ARFAT, Y.A.; AHMED, J.; HIREMATH, N.; AURAS, R.; JOSEPH, A. Thermo-mechanical, rheological, structural and antimicrobial properties of bionanocomposite films based on fish skin gelatin and silver-copper nanoparticles. Food Hydrocolloids, v. 62, p. 191-202, 2017.

ARUMUGAM, G.K.S.; SHARMA, D.; BALAKRISHNAN, R.M.; ETTIYAPPAN, J.B.P. Extraction, optimization and characterization of collagen from sole fish skin. Sustainable Chemistry and Pharmacy, v.9, p.19-26, 2018.

BANDEIRA, S.F. Extração e caracterização da gelatina obtida de cabeça de carpa (Aristichthys mobilis). Orientador: Luiz Antonio de Almeida Pinto, 2009. F. Dissertação de mestrado (Programa de Pós-Graduação em Engenharia e Ciência de Alimentos) - Escola de Química e Alimentos, Universidade Federal do Rio Grande, Rio Grande, RS, Brasil, 2009.

BENJAKUl, S.; KITTIPHATTANABAWON, P. Gelatin. Module in Food Science, 2018. Disponível em: doi:10.1016/b978-0-08-100596-5.21588-6

BKHAIRIA, I.; MHAMDI, S.; JRIDI, M.; NASRI, M. New acidic proteases from Lizaaurataviscera: Characterization and application in gelatin production. International Journal of Biological Macromolecules, v.92, p.533-542, 2016.

BORAN, G.; REGENSTEIN, J.M. Fish gelatin. Advances in food and nutrition research, v.60, p.119-143, 2010.

CASANOVA, F.; MOHAMMADIFAR, M.A.; JAHROMI, M., PETERSEN, H.O.; EYBYE, K.L.; KOBBELGAARD, S.; JESSEN, F. Physico-chemical, structural and techno-functional properties of gelatin from saithe (Pollachius virens) skin. International Journal of Biological Macromolecules, v.156, p.918-927, 2020.

CAI, L.; FENG, J.; REGENSTEIN, J.; LV, Y.; LI, J. Confectionery gels: Effects of low calorie sweeteners on the rheological properties and microstructure of fish gelatin. Food Hydrocolloids, p.67, v.157-165, 2017.

CHUNG, D. Fish gelatin. Biopolymer-Based Formulations, p.67-85, 2020.

CHEUNG, I.W.Y.; LI-CHAN, E.C.Y. Enzymatic production of protein hydrolysates from steelhead (Oncorhynchus mykiss) skin gelatin as inhibitors of dipeptidyl-peptidase IV and angiotensin-I converting enzyme. Journal of Functional Foods, v.28, p.254-264, 2017. 
FAO. 2020. The State of World Fisheries and Aquaculture 2020. Sustainability in action. Rome.

FAUZI, M.B.; LOKANATHAN, Y.; AMINUDDIN, B.S.; RUSZYMAH, B.H.I.; CHOWDHURY, R.S. Ovine tendon collagen: Extraction, characterisation and fabrication of thinfilms for tissue engineering applications. Materials Science and Engineering, v.68, p.163-171, 2016.

GHORANI, B.; EMADZADEH, B.; REZAEINIA, H.; RUSSELL, S.J. Improvements in gelatin cold water solubility after electrospinning and associated physicochemical, functional and rheological properties. Food Hydrocolloids, v.104, p.105740, 2020.

HUANG, T.; TU, Z.; SHANGGUAN, X.; WANG, H.; SHA, X.; BANSAL, N. Rheological behavior, emulsifying properties and structutal characterization of phosphorylated fish gelatin. Food Chemistry, v. 246, p. 428-436, 2018.

HUANG, T.; TU, Z.-C.; WANG, H.; SHANGGUAN, X.; ZHANG, L.; ZHANG, N.-H., BANSAL. Pectin and enzyme complex modified fish scales gelatina: Rheological behavior, gel properties and nanostrusctures. Carbohydrate polymers, v.156, n.535, p.294-302, 2017.

JANGAMREDDY, J.R.; HAAGDORENS, M.K.C.; MIRAZUL ISLAM, M.; LEWIS, P.; SAMANTA, A.; FAGERHOLM, P.; LISZKA, A.; LJUNGGEN, M.K; BUZNYK, O.; ALARCON, E.I.; ZACARIA N.; MEEK, K.M.; GRIFFITH, M. Short peptide analogs as alternatives to collagen in pro-regenerative corneal implants. Acta Biomaterialia, v.69, p.120-130, 2018.

KARAYANNAKIDIS, P.D.; ZOTOS, A. Fish processing by-products as a potential source of gelatin: a review. Journal of Aquatic Food Product Technology, v.25, p.65-92, 2016.

KWATRA, B. Collagen supplementation: therapy for the prevention and treatment of osteoporosis and osteoarthritis: a review. World journal of pharmacy and pharmaceutical sciences, v.9, n.5, p.589-604, 2020.

LAURINDO, E.E.; BARROS, I.R. F. Encefalopatia espongiforme bovina atípica: uma revisão. Arquivo do Instituto Biológico, v.84, p.1-10, 2017.

LIMA-JUNIOR, E.M.; PICOLO, N.S.; MIRANDA, M.J.B.; RIBEIRO, W.L.C.; ALVES, A.P.N.N.; FERREIRA, G.E.; PARENTE, E.A.; MORAES-FILHO, M.O. Uso da pele de tilápia (Oreochromis niloticus), como curativo biológico oclusivo, no tratamento de queimaduras. RevistaBrasileira de Queimaduras, n.16, v. 1, p.10-17, 2017.

LIU, D.; NIKOO, M.; BORAN, G.; ZHOU, P.; REGENSTEIN, J.M. Collagen and gelatin. Annual review of food science and technology, v.6, p.527-557, 2015.

LIU, J.; YONG, H.; LIU, Y.; QIN, Y.; KAN, J.; LIU, J. Preparation and characterization of active and intelligent films based on fish gelatin and haskap berries (Lonicera caerulea L.) extract. Food Packaging and Shelf Life, v.22, artigo 100417, 2019. 
LIU, Y.; LI, B.; ZHANG, K.; LI, J.; HOU, H. Novel hard capsule prepared by tilapia (Oreochromis niloticus) scale gelatin and konjac glucomannan: characterization, and in vitro dissolution. Carbohydrate Polymers, v.206, p.254-261, 2018.

LIN-CHEN, LV.; HUANG, Q.-Y.; DING, W.; XIAO, X.-H.; ZHANG, H.-Y.; XIONG, L.-X. Fish gelatin: The novel potential applications. Journal of Functional Foods, v.63, p.1-14, 2019.

LIN, L.; REGENSTEIN, J.M.; LV, S.; LU, J.; JIANG, S. An overview of gelatin derived from aquatic animals: Properties and modification. Trends in Food Science \& Technology, v.68, p.102-112, 2017.

MIRZAPOUR-KOUHDASHT, A.; MOOSAVI-NASAB， M.; KRISHNASWAMY， K.; KHALESI, M. Optimization of gelatin production from Barred mackerel by-products: Characterization and hydrolysis using native and commercial proteases. Food Hydrocolloids, v.108, p.1-14, 2020.

MIRZAPOUR KOUHDASHT, A.; MOOSAVI-NASAB, M.; AMINLARI, M. Gelatin production using fish wastes by extracted alcalinepretease fromBacillus licheniformis. Journal of food Science and Tecnology, v.1, p.1-6, 2018.

OLIVEIRA, V.M.; CUNHA, M.N.C.; NASCIMENTO, T.P.; ASSIS, C.R.D.; BEZERRA, R.S.; PORTO, A.L.F. Colágeno: características gerais e produção de peptídeos bioativos uma revisão com ênfase nos subprodutos do pescado. Actapesca, v.2, p.70-82, 2017a.

OLIVEIRA, V.M.; CARNEIRO CUNHA, M.N.; ASSIS, C.R.D.; NASCIMENTO, T.P.; HERCUlANO, P. N.; CAVALCANTI, M.T.H.; PORTO, A. L. Colagenases de pescado e suas aplicações industriais. Pubvet, v.11, n.3, p. 243-255, 2017 b.

PANG, Z.; DEETH, H.; YANG, H.; PRAKASH, S.; BANSAL, N. Evaluation of tilapia skin gelatin as a mammalian gelatin replacer in acid Milk gelsand low-fatstirred yogurt. Journal of Dairy Science, v.100, n.5, p.3436-3447, 2017.

PĘPCZYŃSKA, M.; DÍAZ-CALDERÓN, P.; QUERO, F.; MATIACEVICH, S.; CHAR, C.; ENRIONE, J. Interaction and fragility study in salmon gelatin-oligosaccharide composite films at low moisture conditions. Food Hydrocolloids, v.97, p.105-207, 2019.

PORFIRIO, E.; FANARO, G.B. Collagen supplementation as a complementary therapy for the prevention and treatment of osteoporosis and osteoarthritis: a systematic review. Revista Brasileira de Geriatria e Gerontologia, v. 19, n. 1, p. 153-164, 2016.

RENUKA, V.; RAO RAVISHANKAR, C.N.; ZYNUDHEEN, A.A.; BINDU, J.; JOSEPH, T.C. Characterization of gelatin a obtained from unicornlea ther jacket (Aluterus monocerus) and reef cod (Epinephelus diacanthus) skins. LWT- Food Science and Technology, Disponível em: 108586.doi:10.1016/j.lwt.2019.108586

SANTOS, J.P.; ESQUERDO, V.M.; MOURA, C.M.; PINTO, L.A. A. Crosslinking agents effec ton gelatins from carp and tilapia skins and in their biopolymeric films. Colloidsand Surfaces A: Physicochemical and Engineering Aspects, n.539, p.184-191, 2018. 
SILA, A.; MARTINEZ-ALVAREZ, O.; KRICHEN, F.; GÓMEZ-GUILLÉN, M.C.; BOUGATEF, A. Gelatin prepared from Europeaneel (Anguilla anguilla) skin: Physicochemical, textural, viscoelasticand surface properties. Colloidsand Surfaces A: Physicochemical and Engineering Aspects, v.529, p.643-650, 2019.

SILVA, G.C.O.; SILVA. S.S.; CARVALHO, J.W.P.; GUEDES, F.S.; LOSS, R.A. Obtenção e Caracterização físico-química e microbiológica da gelatina de resíduos de matrinxã (Brycon amazonicus) e tambaqui (Colossoma macropomum). Acta of fisheries and aquatic Resources, v.6, n.1, p.74-84, 2018.

SILVA, E.V.C. Otimização das condições de extração da gelatina de pele de peixes amazônicos por diferentes métodos. 2016. 20f. Tese de doutorado (Programa de Pósgraduação em Ciência e Tecnologia de Alimentos) - Instituto de Tecnologia, Universidade Federal do Pará, Belém-Pará, 2016.

SINTHUSAMRAN, S.; BENJAKUL, S.; SWEDLUND, P.J.; HEMAR, Y. Physical and rheological properties of fish gelatin gel as influencedby $\kappa$-carrageenan. Food Bioscience, v.20, p.88-95, 2017.

SINTHUSAMRAN, S.; BENJAKUL, S. Physical, rheological and antioxidant properties of gelatin gel as affected by thein corporationof $\beta$-glucan. Food Hydrocolloids, v.79, p.409$415,2018$.

STOHS, S.J.; KITCHENS, E.K. Nutritional Supplementation in Health and Sports Performance. Nutrition and Enhanced Sports Performance, p.3-9, 2019.

SOW, L.C.; NICOLE CHONG, J.M.; LIAO, Q.X.; YANG, H. Effects of $\kappa$-carrageenanon thestructure and rheological properties of fish gelatin. Journalof Food Engineering, v.239, p.92-103, 2019.

SOW, L.C.; YU TOH, N.Z.; WONG, C.W.; YANG, H. Combination of sodium alginate with tilapia fish gelatin for improved texture properties and nanostructure modification. Food Hydrocolloids, v.94, p.459-467, 2019.

SUI CHIN, S.; HAN LYN, F.; NUR HANANI, Z.A. Effectof Aloe vera (Aloe barbadensis Miller) gel on the physical and functional properties of fish gelatin films as active packaging. Food Packaging and Shelf Life, v.12, p.128-134, 2017.

SULTANA, S.; ALI, M.E.; AHAMAD, M.N.U. Gelatine, collagen, and single cellproteins as a natural and newlyemerging food ingredients. Preparation and Processing of Religious and Cultural Foods, n.1, p.215-239, 2018.

SUN, L.; LI, B.; SONG, W.; SI, L.; HOU, H. Characterization of Pacific cod (Gadus macrocephalus) skin collagen and fabrication of collagen sponge as a good biocompatible biomedical material. Process Biochemistry, v.63, p.229-235, 2019.

TAN, C.-C.; KARIM, A.A.; UTHUMPORN, U.; GHAZALI, Farid C. Effect extraction temperature on the emulsify ing properties of gelatin from Black tilapia (Oreochromis mossambicus) skin. Food Hydrocolloids,v.108, Artigo106024, 2020. 
TAYEL, A.A.; GHANEM, R.A.; MOUSSA, S.H.; FAHMI, M.; TARJAM, H.M.; ISMAIL, N. Skin protectant textileslo aded with fish collagen, chitosan and oakgalls extract composite. International Journal of Biological Macromolecules, v.117, p.25-29, 2018.

TKACZEWSKA, J.; BUKOWSKI, M.; MAK, P. Identification of Antioxidant Peptides in Enzymatic Hydrolysates of Carp (CyprinusCarpio) Skin Gelatin. Molecules, v.24, n.97, p.1$15,2019$.

ZHANG, Q.; WANG, Q.; LV, S.; LU, J.; JIANG, S.; REGENSTEIN, J.M.; LIN, L. Comparison of collagen and gelatin extracted from the skins of Niletilapia (Oreochromis niloticus) and channel catfish (Ictalurus punctatus). Food Bioscience, v.13, p.41-48, 2016.

ZHANG, T.; TU, Z.; ZOU, Z.; SHANGGUAN, X.; WANG, H.; BANSAL, N. Glycosylated fish gelatin emulsion: Rheological, tribological properties and its application as model coffee creamers. Food Hydrocolloids, v. 102, 2020.

ZHANG, Z.; ZHU, H.; ZHENG, Y.; ZHANG, L.; WANG, X.; LUO, Z.; TANG, J.; LIN, L.; DU, Z.; DONG, C. The effects and mechanism of collagen peptide and elastin peptide on skin aginginduced by D-galactose combined with ultraviolet radiation. Journal of Photochemistry and Photobiology B: Biology, v.210, p.1-8, 2020.

ZHANG, T.; DING, M.; TAO, L.; LIU, L.; TAO, N.; WANG, X.; ZHONG, J. Octenyl succinic anhydride modification of bovine bone and fish skin gelatins and their application for fishoil-loadedemulsions. Food Hydrocolloids, v.108, p.1-10, 2020.

ZHANG, T.; SUN, R.; DING, M.; LI, L.; TAO, N.; WANG, X.; ZHONG, J. Commercial cold-water fish skin gelatin and bovine bone gelatina: structural, functional, andemulsionstability diferences. LWT- Food Science and Technology, v.125, 2020.

YOON, H.J.; SHIN, S.R.; CHA, J.M.; LEE, S.-H.; KIM, J.-H.; DO, J.T.; BAE, H. Cold Water Fish Gelatin Methacryloyl Hydrogel for Tissue Engineering Application.PLoS ONE,v.11 n. 10, Artigo e0163902, 2016. 